\title{
Design of Array of Rectangular Superconducting Microstrips with Multilayer Dielectrics
}

\author{
O. Barkat, and M. Bensalem
}

\begin{abstract}
The analysis of array of rectangular superconducting microstrips implemented in multilayered substrates is presented in this research. The spectral domain and the stationary phase formulations of single rectangular superconducting microstrip antenna are given, in order to calculate its resonant frequencies and its radiation patterns. Additionally, the swarm particle optimization (PSO) is employed for the synthesis of linear array geometry. The main objective of this optimization is to achieve minimum side lobe levels, and maximum directivity. Then, we calculate and plot the total radiated field which is the multiplication of a single element radiation pattern by the array factor, when superconducting rectangular microstrip antennas are made in a linear arrangement. Comparing to the exiting array antenna design, the numerical results, obtained from the implementation of our calculations, have shown that the optimum parameters of amplitudes of excitation and positions obtained by our formulation using PSO increases the gain, and improve the directivity.
\end{abstract}

Keywords - Antennas arrays, Full wave, Multilayer dielectric, Superconducting rectangular, Swarm particle.

\section{INTRODUCTION}

Microstrip antennas have received increasing attention invarious communication systems applications. These antennas are extremely popular for use in the range of microwave frequencies. One of the major advantages of microstrip antennas is the simplicity of array construction [1-3]. Usually, the radiation pattern of a single element is relatively wide, and each element provides low values of directivity. In many applications, it is necessary to design antennas with very directive characteristics. This can only be accomplished by grouping multiple radiating elements to form an antenna array. The microstrip antennas in linear array may be square, rectangular, circular, elliptical, triangular, and any other configuration. It may regular or irregular. The printed array antennas are constituted by radiating elements connected to each other by sections of micro-strip line.

The rectangular microstrip is the simplest configuration since it is the easiest to analyze mathematically. The efficiency of microstrip antenna arrays may be improved significantly by reducing losses in the feed network. Losses in the microstrip

O. Barkat Department of Electronics, Electromagnetism and Telecommunications Laboratory, University Frères Mentouri Constantine 1, Algeria.

M. Bensalem, Department of Electronics, Electromagnetism and Telecommunications Laboratory, University Frères Mentouri Constantine 1, Algeria. feed network are due to conductor loss, radiation loss and dielectric loss. Thus, most antennas are fabricated from low loss metals such as copper or gold. It is also quite common to use superconducting antennas which have very low losses up to frequencies up to a few Gigahertz corresponding to the energy gap of the superconductor [4-6]. An increasing use of multilayer planar structures in the development of many microwave devices is one of the major trends in the microwave area.

The layers of suitable dielectrics may be used to enhance the directivity, also are useful to provide protection to patch from heat, rain and physical damage, wide bandwidth and high gain. In recent years, several methods have been proposed for the calculation of resonant frequencies and far field radiation patterns of rectangular microstrip antennas. Many of these models employ approximations that are valid only for thin substrates. Among these methods we can cite: the transmission-line model and the cavity model. These models usually treat the microstrip patch as a transmission line or as a cavity resonator. The full-wave method, also known as the method of moments, is arguably the most popular method for the analysis of microstrip antennas. This is because of their applicability to determine the resonant characteristics of high critical temperature $(\mathrm{Tc})$ superconducting microstrip antennas [7].

Several studies have been proposed and conducted for optimization of characteristics of linear array of antennas. The main goal of optimization is to find the best parameters valuesthat maximize or minimize all objective functions. Particle swarm optimization (PSO) and genetic algorithms (GA) are two popular classes of evolutionary algorithms that have been applied successfully in antenna arrays. We can learn that PSO shares many similarities with GA. Both algorithms begin with a set of randomly generated population and each algorithm has fitness values to evaluate the population, update the population and search for the optimum with random techniques. On the other hand, PSO does not have genetic operators like crossover and mutation. Particles update themselves with the internal velocity. Also, many studies show that PSO algorithm convergence is faster than GA.

This work will describe analysis and synthesis of array of rectangular superconducting microstrips with multilayer dielectrics. The radiation characteristics of single superconducting rectangular microstrip, which is integrated on anisotropic layers substrates, are investigated using a Fourier transforms domain in conjunction with the stationary phase 
Design of Array of Rectangular Superconducting Microstrips with Multilayer Dielectrics O. Barkat, and M. Bensalem Department of Electronics, Electromagnetism and Telecommunications Laboratory, University Frères Mentouri Constantine 1, Algeria method. For optimization of linear array of High Tc superconducting rectangular microstrip antennas, we used the particle swarm optimization (PSO); this method develops a novel objective function to incorporate with a PSO for solving the resulting optimization problem.

\section{ANALYSIS AND OPTIMIZATION}

The array of high Tc superconducting microstrips mounted in multilayer dielectrics considered is shown in Figure 1. The superconducting rectangular patch of thickness $t$, with length a and width $b$. The plan $\mathrm{Mz}$ is the rectangular patches interface. Each layer $\mathrm{j}$ is characterized by thickness $d \mathrm{j}=\mathrm{zj}-\mathrm{zj}-1$, and a complex dielectric permittivity $\mathrm{j} e$, the permeability is everywhere assumed to be $\mathrm{m} 0$. In order to find the dyadic Green's function of the structure, the calculus of transverse field components in the jth layer is the process starts. We will suppose that a space time dependence of all the components of the kind $\exp (\mathrm{i}(\mathrm{k} \cdot \mathrm{r}-\mathrm{wt}))$.

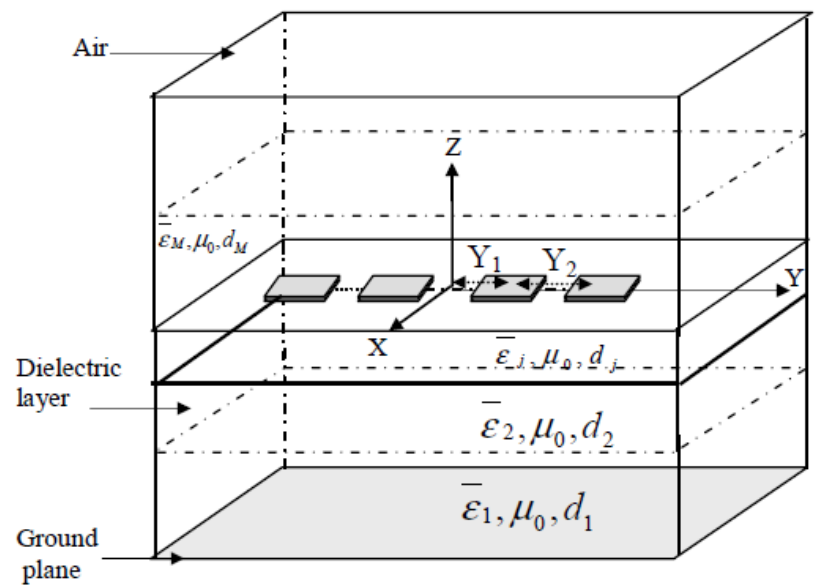

Fig. 1. Geometry of $\mathrm{N}$ element non periodic linear array with multilayer dielectrics

The transverse field components by using the Fourier transform formulation can be given by [8]:

$$
\begin{gathered}
\widetilde{\widetilde{E}}\left(k_{s}, z\right)=\int_{-\infty}^{+\infty} \int_{-\infty}^{+\infty} \bar{E}(x, y, z) \cdot e^{-j\left(k_{x} x+k_{y} \cdot y\right)} d x \cdot d y \\
\widetilde{\bar{H}}\left(k_{s}, z\right)=\int_{-\infty-\infty}^{+\infty} \int_{-\infty} \bar{H}(x, y, z) \cdot e^{-j\left(k_{x} \cdot x+k_{y} \cdot y\right)} d x \cdot d y
\end{gathered}
$$

By using Maxwell's equations in the Fourier transform domain, we can show that the transverse fields inside the jth layer can be written as:

$$
\widetilde{\bar{E}}\left(k_{s}, z\right)=\left[\begin{array}{l}
\widetilde{E}_{x}\left(k_{s}, z\right) \\
\widetilde{E}_{y}\left(k_{s}, z\right)
\end{array}\right]=\frac{1}{k_{s}^{2}}\left[\begin{array}{l}
\frac{i k_{x} \varepsilon_{z}}{\varepsilon_{x}} \frac{\partial \widetilde{E}_{z}\left(k_{s}, z\right)}{\partial z}+k_{y} w \mu_{0} \widetilde{H}_{z}\left(k_{s}, z\right) \\
\frac{i k_{Y} \varepsilon_{z}}{\varepsilon_{x}} \frac{\partial \widetilde{E}_{z}\left(k_{s}, z\right)}{\partial z}-k_{x} w \mu_{0} \widetilde{H}_{z}\left(k_{s}, z\right)
\end{array}\right]
$$

$$
\tilde{\tilde{H}}\left(k_{s}, z\right)=\left[\begin{array}{c}
\widetilde{H}_{y}\left(k_{s}, z\right) \\
-\widetilde{H}_{x}\left(k_{s}, z\right)
\end{array}\right]=\frac{1}{k_{s}^{2}}\left[\begin{array}{l}
i k_{x} w \varepsilon_{0} \varepsilon_{z} \widetilde{E}_{z}\left(k_{s}, z\right)+i k_{y} \frac{\partial \widetilde{H}_{z}\left(k_{s}, z\right)}{\partial z} \\
k_{y} w \varepsilon_{0} \varepsilon_{z} \widetilde{E}_{z}\left(k_{s}, z\right)-i k_{x} \frac{\partial \tilde{H}_{z}\left(k_{s}, z\right)}{\partial z}
\end{array}\right]
$$

This can also be written as:

$$
\begin{gathered}
\widetilde{e}=\left[\begin{array}{l}
\widetilde{e}^{e} \\
\widetilde{e}^{h}
\end{array}\right]=\left[\begin{array}{ll}
\frac{i \varepsilon_{z}}{k_{s} \varepsilon_{x}} & \frac{\partial \widetilde{E}_{z}}{\partial z} \\
\frac{w \mu_{0}}{k_{s}} & \widetilde{H}_{z}
\end{array}\right] \\
\widetilde{\bar{h}}=\left[\begin{array}{c}
\widetilde{h}^{e} \\
\widetilde{h}^{h}
\end{array}\right]=\left[\begin{array}{l}
\frac{w \varepsilon_{0} \varepsilon_{z}}{k_{s}} \\
\widetilde{E}_{z} \\
i \frac{\partial \widetilde{H}_{z}}{k_{s} \partial z}
\end{array}\right]
\end{gathered}
$$

Where

$$
\begin{aligned}
& \widetilde{\mathrm{E}}_{z}\left(\mathrm{k}_{\mathrm{s}}, \mathrm{z}\right)=\mathrm{A}_{\mathrm{j}}^{\mathrm{e}} \mathrm{e}^{-\mathrm{i} \mathrm{k}_{z}^{\mathrm{e} z}}+\mathrm{B}_{\mathrm{j}}^{\mathrm{e}} \mathrm{e}^{\mathrm{i} \mathrm{k}_{z}^{\mathrm{e} z} z} \\
& \widetilde{\mathrm{H}}_{z}\left(\mathrm{k}_{\mathrm{s}}, \mathrm{z}\right)=\mathrm{A}_{\mathrm{j}}^{\mathrm{h}} \mathrm{e}^{-\mathrm{i} \mathrm{k}_{\mathrm{g} z}^{\mathrm{h} z}}+\mathrm{B}_{\mathrm{j}}^{\mathrm{h}} \mathrm{e}^{\mathrm{i} \mathrm{k}_{z}^{\mathrm{h} z}}
\end{aligned}
$$

In which

$$
\begin{aligned}
& \mathrm{k}_{\mathrm{zj}}^{\mathrm{e}}=\left(\varepsilon_{\mathrm{xj}} \mathrm{k}_{0}^{2}-\frac{\varepsilon_{\mathrm{xj}}}{\varepsilon_{\mathrm{zj}}} \mathrm{k}_{\mathrm{s}}^{2}\right)^{\frac{1}{2}} \\
& \mathrm{k}_{\mathrm{zj}}^{\mathrm{h}}=\left(\varepsilon_{\mathrm{xj}} \mathrm{k}_{0}^{2}-\mathrm{k}_{\mathrm{s}}^{2}\right)^{\frac{1}{2}} \\
& k_{0}=\omega \sqrt{\mu_{0} \varepsilon_{0}}
\end{aligned}
$$

$A_{j}^{e}, B_{j}^{e}, A_{j}^{h}$ et $B_{j}^{h}$ are unknown vectors. Eliminating these unknowns in (7) and (8), a relation is obtained for the components $\widetilde{\mathrm{e}}$ and $\widetilde{\overline{\mathrm{h}}}$ at the interfaces of the layer, is given by relation (9).

$$
\left[\begin{array}{l}
\stackrel{-}{\mathbf{e}}\left(k_{s}, z_{j}^{-}\right) \\
\tilde{\mathbf{h}}\left(k_{s}, z_{j}^{-}\right)
\end{array}\right]=\bar{M}_{j} \cdot\left[\begin{array}{l}
\widetilde{\mathbf{e}}\left(k_{s}, z_{j-1}^{+}\right) \\
\widetilde{\mathbf{h}}\left(k_{s}, z_{j-1}^{+}\right)
\end{array}\right]
$$

With

$$
\bar{M}_{j}=\left[\begin{array}{cc}
\bar{I} \cos \left(\bar{k}_{z j} d_{j}\right) & -i \bar{g}_{j}^{-1}\left(k_{s}\right) \sin \left(\bar{k}_{z z} d_{j}\right) \\
-i \bar{g}_{j}\left(k_{s}\right) \sin \left(\bar{k}_{z j} d_{j}\right) & \bar{I} \cos \left(\bar{k}_{z j} d_{j}\right)
\end{array}\right]
$$

Where $\overline{\mathrm{I}}$ being the $(2 \mathrm{x} 2)$ unit matrix and $\overline{\mathrm{k}}_{\mathrm{zj}}$ and $\overline{\mathrm{g}}_{\mathrm{j}}$ are given by:

$$
\overline{\mathrm{k}}_{\mathrm{zj}}=\left|\begin{array}{cc}
\mathrm{k}_{\mathrm{zj}}^{\mathrm{e}} & 0 \\
0 & \mathrm{k}_{\mathrm{zj}}^{\mathrm{h}}
\end{array}\right| \text { and } \quad \bar{g}_{j}=\left|\begin{array}{cc}
\frac{\omega \varepsilon_{0} \varepsilon_{x j}}{k_{z j}^{e}} & 0 \\
0 & \frac{k_{z j}^{h}}{\omega \mu_{0}}
\end{array}\right|
$$

The relation between the tangential electric and magnetic fields components in dielectric layers below the patches interface is given by: 


$$
\left[\begin{array}{l}
\widetilde{\tilde{\mathbf{e}}}\left(k_{s}, z_{M}^{-}\right) \\
\widetilde{\sim}\left(k_{s}, z_{M}^{-}\right)
\end{array}\right]=\bar{M}_{1} \cdot \bar{M}_{2} \cdot \bar{M}_{3} \ldots . \bar{M}_{M} \cdot\left[\begin{array}{c}
\widetilde{\tilde{\mathbf{e}}}\left(k_{s}, 0\right) \\
\widetilde{\mathbf{h}}\left(k_{s}, 0\right)
\end{array}\right]
$$

Above the patches interface, the relation between the tangential electric and magnetic fields components in dielectric layers is given by:

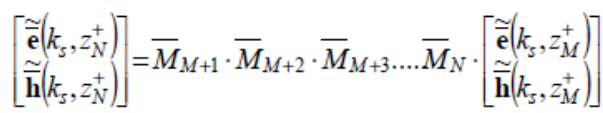

We can put the product matrix as:

$$
\overline{\boldsymbol{\Gamma}}_{<}=\prod_{j=M}^{1} \bar{M}_{j}=\left[\begin{array}{cc}
\Gamma_{\prec}^{11} & \Gamma_{\prec}^{12} \\
\Gamma_{\prec}^{21} & \Gamma_{\prec}^{22}
\end{array}\right]
$$

and

$$
\overline{\boldsymbol{\Gamma}}_{\succ}=\prod_{j=N}^{M+1} \bar{M}_{j}=\left[\begin{array}{ll}
\Gamma_{\succ}^{11} & \Gamma_{\succ}^{12} \\
\Gamma_{\succ}^{21} & \Gamma_{\succ}^{22}
\end{array}\right]
$$

Where $\prod_{j=M}^{1}$ and $\prod_{j=N}^{M+1}$ means the multiplication of matrices above and below the patches.

By using the boundary conditions and condition of continuity of $\mathrm{E}$ and $\mathrm{H}$ fields, we can find that the relationship between the patch current and the electric field on the patch is given by [8]:

$$
\widetilde{\overline{\mathrm{E}}}\left(\mathrm{k}_{\mathrm{s}}\right)=\overline{\mathrm{G}}\left(\mathrm{k}_{\mathrm{s}}\right) \cdot \widetilde{\widetilde{\mathrm{J}}}\left(\mathrm{k}_{\mathrm{s}}\right)
$$

Where $\widetilde{J}\left(k_{s}\right)$ is the current on the patch, and $\bar{G}$ is the spectral dyadic Green's function, its expression is shown to be given by:

$$
\bar{G}\left(k_{s}\right)=\left[\begin{array}{ll}
G_{x x} & G_{x y} \\
G_{y x} & G_{y y}
\end{array}\right]=\frac{1}{k_{s}^{2}} \cdot\left[\begin{array}{cc}
k_{x} & k_{y} \\
k_{y} & -k_{x}
\end{array}\right] \cdot \bar{Q} \cdot\left[\begin{array}{cc}
k_{x} & k_{y} \\
k_{y} & -k_{x}
\end{array}\right]
$$

Where $Q$ is given by:

$$
Q\left(k_{s}\right)=-\left(\overline{\boldsymbol{\Gamma}}_{<}\right)_{12} \cdot\left[\mathbf{\underline { \mathbf { g } }}_{0} \cdot\left(\overline{\boldsymbol{\Gamma}}_{>}\right)_{12}-\left(\overline{\boldsymbol{\Gamma}}_{>}\right)_{22}\right] \cdot\left[\overline{\mathbf{g}}_{0} \cdot(\overline{\boldsymbol{\Gamma}})_{12}-(\overline{\boldsymbol{\Gamma}})_{22}\right]^{-1}
$$

The surface current $\overline{\mathrm{J}}\left(\mathrm{r}_{\mathrm{s}}\right)$ on the patch can be expanded into a finite series of known basis functions $J_{x n}$ and and $J_{y n}$. [9]:

$$
\overline{\mathrm{J}}\left(\mathbf{r}_{s}\right)=\sum_{n=1}^{N} a_{n}\left[\begin{array}{c}
J_{x n}\left(\mathbf{r}_{s}\right) \\
0
\end{array}\right]+\sum_{m=1}^{M} b_{m}\left[\begin{array}{c}
0 \\
J_{y m}\left(\mathbf{r}_{s}\right)
\end{array}\right]
$$

Where an and bn are the mode expansion coefficients to be determined in the $\mathrm{x}$ and $\mathrm{y}$ direction respectively.

The main problem is how to select the basis functions associated with the complete orthogonal set of TM and TE modes of rectangular microstrip antenna (figure 2).

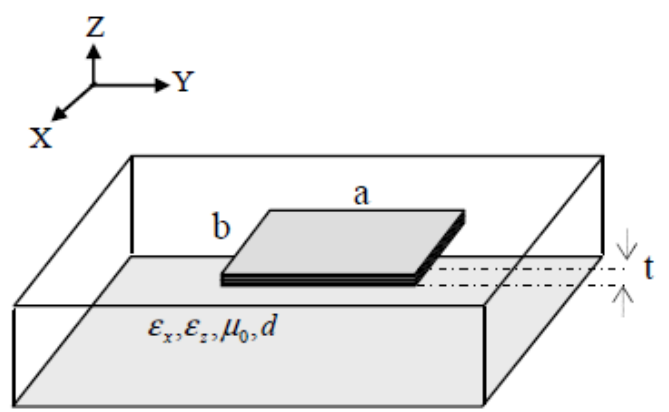

Fig. 2. Geometry of High superconducting rectangular microstrip on uniaxial substrate

To include the effect of the superconductivity of the microstrip antenna in full-wave analysis, the impedance is determined by using London's equation and the model of Gorter and Gasimir, it is given by [5]:

$$
\mathrm{Z}_{\mathrm{s}}=\mathrm{R}_{\mathrm{s}}+\mathrm{iX} \mathrm{s}
$$

Where $\mathrm{Zs}$ is the surface impedance of the superconducting patch, Rs and Xs are the surface resistance and the surface reactance.

When the thickness $(\mathrm{t})$ of the superconducting patch is less than three times the zero-temperature penetration depth ( 01 ), the surface impedance can be approximated as follows:

$$
Z_{S}=\frac{1}{t \sigma}
$$

Where $\sigma$ is the complex conductivity of the superconducting film, is given by

$$
\sigma=\sigma_{1}-i \sigma_{2}
$$

The conductivity components are given by: $\sigma_{1}=\sigma_{n}\left(T / T_{c}\right)^{4}$ and $\sigma_{2}=\frac{1-\left(T / T_{c}\right)^{4}}{\omega \mu_{0} \lambda_{0}^{2}}$.

Where $\sigma_{n}$ : Is the normal state conductivity at $\mathrm{T}=\mathrm{T}_{\mathrm{C}}$, and $\omega$ is the angular frequency.

Enforcement of the boundary conditions requiring that the transverse component of the electric field to vanish on the High superconducting rectangular microstrip, and the current on the patch is then formulated using the dyadic Green's function.

$$
\overline{\mathrm{E}}\left(\mathrm{r}_{\mathrm{s}}\right)=\frac{1}{(2 \pi)^{2}} \int \mathrm{dk}_{\mathrm{s}} \cdot \mathrm{F}\left(\mathrm{k}_{\mathrm{s}}, \mathrm{r}_{\mathrm{s}}\right) \cdot\left(\overline{\mathrm{G}}\left(\mathrm{k}_{\mathrm{s}}\right)-\overline{\mathrm{Z}}_{\mathrm{s}}\right) \cdot \widetilde{\mathrm{J}}\left(\mathrm{k}_{\mathrm{s}}\right)=0
$$

Where $\bar{Z}_{\mathrm{s}}=\left|\begin{array}{cc}Z_{\mathrm{s}} & 0 \\ 0 & Z_{\mathrm{s}}\end{array}\right|$

Substituting (16) into (22) and using the selected basis functions as testing functions, we obtain the following homogeneous matrix equation:

$$
\left[\begin{array}{ll}
{\left[\overline{\mathrm{B}}_{\mathrm{kn}}\right]_{\mathrm{NxN}}} & {\left[\overline{\mathrm{B}}_{\mathrm{km}}\right]_{\mathrm{NxM}}} \\
{\left[\overline{\mathrm{B}}_{\mathrm{ln}}\right]_{\mathrm{MxN}}} & {\left[\overline{\mathrm{B}}_{\mathrm{lm}}\right]_{\mathrm{MxM}}}
\end{array}\right] \cdot\left[\begin{array}{c}
{\left[\mathrm{a}_{\mathrm{n}}\right]_{\mathrm{NX} 1}} \\
{\left[\mathrm{~b}_{\mathrm{m}}\right]_{\mathrm{MX} 1}}
\end{array}\right]=\left|\begin{array}{l}
{[0]_{\mathrm{NX} 1}} \\
{[0]_{\mathrm{MX} 1}}
\end{array}\right|
$$

Where 


$$
\begin{gathered}
\overline{\mathrm{B}}_{\mathrm{kn}}=\int_{-\infty}^{+\infty} \int_{-\infty}^{+\infty} \mathrm{dk}_{\mathrm{s}} \frac{1}{\mathrm{k}_{\mathrm{s}}^{2}}\left(\mathrm{G}_{\mathrm{xx}}-\mathrm{Z}_{\mathrm{s}}\right) \widetilde{\mathrm{J}}_{\mathrm{xk}}\left(-\mathrm{k}_{\mathrm{s}}\right) \widetilde{\mathrm{J}}_{\mathrm{xm}}\left(\mathrm{k}_{\mathrm{s}}\right) \\
\overline{\mathrm{B}}_{\mathrm{km}}=\int_{-\infty}^{+\infty} \int_{-\infty}^{+\infty} \mathrm{dk}_{\mathrm{s}} \frac{\mathrm{k}_{\mathrm{x}} \mathrm{k}_{\mathrm{y}}}{\mathrm{k}_{\mathrm{s}}^{2}} \mathrm{G}_{\mathrm{xy}} \widetilde{\mathrm{J}}_{\mathrm{xk}}\left(-\mathrm{k}_{\mathrm{s}}\right) \widetilde{\mathrm{J}}_{\mathrm{ym}}\left(\mathrm{k}_{\mathrm{s}}\right) \\
\overline{\mathrm{B}}_{\mathrm{ln}}=\int_{-\infty}^{+\infty} \int_{-\infty}^{+\infty} \mathrm{dk}_{\mathrm{s}} \frac{\mathrm{k}_{\mathrm{x}} \mathrm{k}_{\mathrm{y}}}{\mathrm{k}_{\mathrm{s}}^{2}} \mathrm{G}_{\mathrm{ys}} \widetilde{\mathrm{J}}_{\mathrm{yl}}\left(-\mathrm{k}_{\mathrm{s}}\right) \widetilde{\mathrm{J}}_{\mathrm{xn}}\left(\mathrm{k}_{\mathrm{s}}\right) \\
\overline{\mathrm{B}}_{\mathrm{lm}}=\int_{-\infty}^{+\infty} \int_{-\infty}^{+\infty} \mathrm{dk}_{\mathrm{s}} \frac{1}{\mathrm{k}_{\mathrm{s}}^{2}}\left(\mathrm{G}_{\mathrm{yy}}-\mathrm{Z}_{\mathrm{s}}\right) \widetilde{\mathrm{J}}_{\mathrm{yl}}\left(-\mathrm{k}_{\mathrm{s}}\right) \widetilde{\mathrm{J}}_{\mathrm{ym}}\left(\mathrm{k}_{\mathrm{s}}\right)
\end{gathered}
$$

Therefore, for the existence of nontrivial solutions, the determinant of (31) must be zero.

$$
\operatorname{det}(\overline{\mathrm{B}}(\mathrm{f}))=0
$$

In general the root of equation (28) is the characteristic equation for the complex resonant frequency $f=f_{r}+i f_{i}$.

$f_{r}$ is the resonant frequency and $2 f_{i} / f_{r}$ is the half-power bandwidth of the antenna.

Once the problem is solved for the resonant frequency, far field radiations in spherical coordinates are given from the vector Fourier transform of the electric field.

$$
\begin{gathered}
E_{x}(x, y)=\frac{1}{4 \cdot \pi^{2}} \int_{-\infty}^{+\infty} \int_{-\infty}^{+\infty}\left[\left(G_{x x}-Z_{S}\right) \cdot \widetilde{J}_{x}+G_{x y} \widetilde{J}_{y}\right] e^{j\left(k_{x} \cdot x+k_{y} \cdot y\right)} \cdot d k_{x} \cdot d k_{y} \\
E_{y}(x, y)=\frac{1}{4 \cdot \pi^{2}} \int_{-\infty}^{+\infty} \int_{-\infty}^{+\infty}\left[G_{y x} \cdot \widetilde{J}_{x}+\left(G_{y y}-Z_{S}\right) \cdot \widetilde{J}_{y}\right] \cdot e^{j\left(k_{x} \cdot x+k_{y} \cdot y\right)} \cdot d k_{x} \cdot d k_{y}
\end{gathered}
$$

Using the stationary phase method, we can obtain the farzone, co-polar and cross- polar field components of structure are, respectively given by:

$$
\begin{gathered}
E_{\phi}(\theta, \phi)=-E_{x} \sin \phi+E_{y} \cos \phi \\
E_{\theta}(\theta, \phi)=E_{x} \cos \theta \cos \phi+E_{y} \cos \theta \sin \phi
\end{gathered}
$$

The electric field component are defined in the interval

$$
0 \leq \theta \leq \pi, \quad 0 \leq \phi \leq 2 \pi
$$

An array has many advantages over a single element. A linear array has excellent directivity and it can form the narrowest main-lobe in a given direction [10]-[12]. The total far field of any antenna array with identical radiating elements is the product of the element factor $\mathrm{E} \Theta, \phi$ and array factor $\mathrm{AF}$. The $\mathrm{AF}$ depends on the geometric arrangement of the array elements, the spacing of the elements, and excitation of each element. By varying the separation $\mathrm{Y}$ and/or the phase $\psi$ between the elements, the characteristics of the array factor and the total field of the array can be controlled.

$$
F(\theta, \phi)=E_{\theta, \Phi} \cdot A F(\theta, \phi)
$$

Where

$$
\mathrm{AF}(\theta, \phi)=\sum_{\mathrm{k}=1}^{2 \mathrm{~N}} \mathrm{~A}_{\mathrm{k}} \exp \left(\mathrm{i}\left(\mathrm{k}_{0} \mathrm{Y}_{\mathrm{k}} \sin \theta \cos \phi+\psi_{\mathrm{k}}\right)\right)
$$

The Excitation coefficients (amplitude and phase) of the $\mathrm{N}$ elements are chosen to be: ${ }^{I_{k}}=A_{k} e^{j \psi_{k}}$. Through, to determine the values of parameters ( $Y_{k}$ : distance, and $A_{k}$ : amplitude), we used particle swarm optimization (PSO) to approximate the radiation pattern function, for getting better the radiation pattern of array. Particle swarm optimization (PSO) was originally developed by Dr. Eberhart and Dr. Kennedy in 1995, inspired by social behavior of swarms. It has been shown to have excellent abilities in optimizing multi-dimensional and multi-objective problems. The basic pseudo code for the PSO algorithm, given by [13].

In order to solve any problem with PSO, we need to define a fitness function which will be used to measure the quality of possible solutions for that problem. In antenna array problems, there are many parameters that can be used to evaluate function such as gain, side lobe level, and radiation pattern. Here, we are interested in designing a linear antenna array with minimum side lobes levels. Thus, the following fitness function is applied to the evaluation, defined as:

$$
\text { fitness }=\min \left(\sum_{\varphi_{i}=\varphi_{0}}^{\varphi_{i}=\varphi_{R}}\left(\max \operatorname{SLL}\left(\theta, \varphi_{i}\right)\right) / R\right)
$$

Where

$\max$ SLL : is the maximum sidelobe level of linear antenna array of $2 \mathrm{~N}$ isotropic elements obtained by using PSO.

$\mathrm{R}$ : is the total sample point.

$\phi_{i}$ : are points where we have maximum sidelobe level

$\phi_{0}$ : is initial value of azimuthal angle

$\phi_{\mathrm{R}}$ : is maximal value of azimuthal angle

\section{NumericAl ReSUlts AND DisCUSSION}

In order to investigate the accuracy of the theoretical formulation in previous section, we compared our results concerning superconducting rectangular microstrip antenna. Table 1 demonstrated relation between the real part of frequency resonance, bandwidth, and the thickness of superconducting film $t$ of the antenna, is considered for four different relative permittivity of substrate. The superconducting material (YBCO) characteristics are: $\sigma \mathrm{n}=210 \mathrm{~s} / \mathrm{mm}, 10=1500 \AA$ and $\mathrm{T} / \mathrm{Tc}=0.5, \mathrm{a}=1630 \mu \mathrm{m}, \mathrm{b}=935 \mu \mathrm{m}, \mathrm{d}=254 \mu \mathrm{m}$. In table 1 , it is observed that as the thickness of superconductor patch grows, the resonant frequency increases. These behaviors agree with those reported by Richard and all [4]. Also the numerical results show that, the antenna parameters (resonant frequency and bandwidth) do not vary significantly with the permittivity variation perpendicular to the optical axis (ex). Moreover, these are found to be strongly dependent with the permittivity (ez) is changed and (e1x) remains constant. 
TABLE I: FREQUENCY RESONANT AND BANDWIDTH OF VARIOUS SUPERCONDUCTING RECTANGULAR MICROSTRIP

\begin{tabular}{|c|c|c|c|c|}
\hline$\varepsilon_{\mathrm{x}}$ & $\varepsilon z$ & $\mathrm{t}(\mu \mathrm{m})$ & $\begin{array}{l}\text { Resonant } \\
\text { Frequency } \\
(\mathrm{GHz})\end{array}$ & Bandwidth (\%) \\
\hline \multirow[t]{3}{*}{9,4} & 11.6 & 0.02 & 41.28 & 5.42 \\
\hline & & 0.08 & 41.36 & 5.37 \\
\hline & & 0.1 & 41.37 & 5.35 \\
\hline \multirow[t]{3}{*}{11.6} & 11.6 & 0.02 & 40.54 & 7.87 \\
\hline & & 0.08 & 40.61 & 7.76 \\
\hline & & 0.1 & 40.62 & 7.74 \\
\hline \multirow[t]{3}{*}{13} & 10.3 & 0.02 & 42.75 & 12.33 \\
\hline & & 0.08 & 42.81 & 12.21 \\
\hline & & 0.1 & 42.81 & 12.20 \\
\hline \multirow[t]{3}{*}{10.3} & 10.3 & 0.02 & 42.22 & 10.41 \\
\hline & & 0.08 & 42.91 & 09.12 \\
\hline & & 0.1 & 42.942 & 09.04 \\
\hline
\end{tabular}

Table 2 summarizes the computed resonant frequencies for various perfectly rectangular microstrip. We have a perfectly conducting rectangular antenna, is printed on a substrate of permittivity e $1 \mathrm{x}=\mathrm{e} 1 \mathrm{z}=2.33$, with a thickness $\mathrm{d}=0.3175 \mathrm{~cm}$. The air gap has the effect of reducing the effective permittivity of the cavity beneath the patch, resulting rising shift in the resonant frequency. We compare our theoretical values for three different values of $a$ and $b$, by using the full-wave moment method (MoM), with experimental data, which have been suggested in [14], note that the agreement is very good.

TABLE II: FREQUENCY RESONANT OF VARIOUS PERFECTLY RECTANGULAR MICROSTRIP ANTENNAS

\begin{tabular}{|c|c|c|c|c|}
\hline \multirow[t]{2}{*}{$\mathrm{a}(\mathrm{cm})$} & \multirow[t]{2}{*}{$\mathrm{b}(\mathrm{cm})$} & \multirow[t]{2}{*}{$\mathrm{h}(\mathrm{mm})$} & \multicolumn{2}{|c|}{ "Resonant frequencies (GHz) } \\
\hline & & & Computed [11] & Our results \\
\hline 5.70 & 3.8 & 0.5 & 2.51 & 2.426 \\
\hline 4.55 & 3.05 & 0.5 & 3.05 & 2.976 \\
\hline 2.95 & 1.95 & 0.5 & 4.42 & 4.49 \\
\hline
\end{tabular}

We consider reducing the sidelobe level in a linear array of the 8,10 and 16 isotropic elements, which are non-uniform spacing and excitations of elements. In order to illustrate the capabilities of the particle swarm optimization (PSO) to find acceptable side lobe level (SLL), equal or less than the desired radiation pattern

The PSO program has been written in Matlab language, using 300 iterations. The number of particles taken is 200 , the values of $\mathrm{C} 1$ and $\mathrm{C} 2$ are 2 respectively, and we have set $(\mathrm{W} 1=0.9, \mathrm{~W} 2$ $=0.4$ ). The minimum of fitness function values against number of generation is demonstrated in figure 3, for even number of elements positioned symmetrically along the $\mathrm{x}$-axis. From this figure, it is clear that fitness function converges to optimal solution.

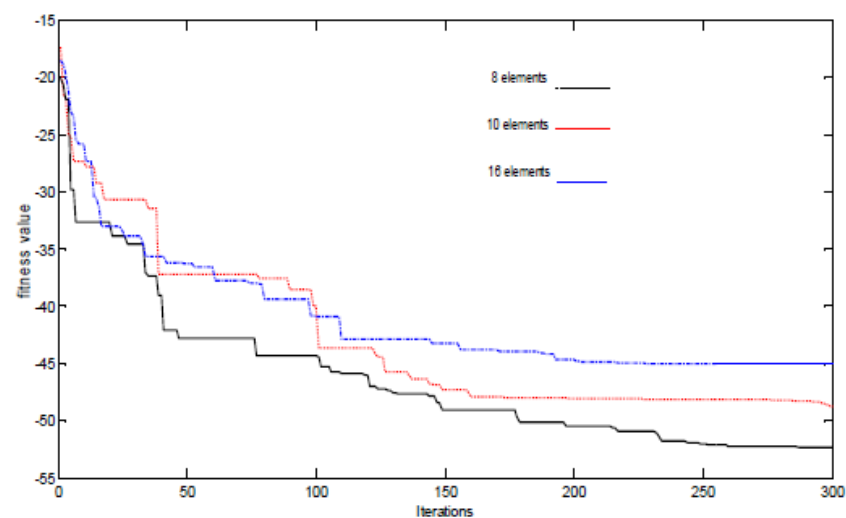

Fig. 3. Convergence curve of the fitness value of the 8,10 and 16 elements linear array versus the number of iterations

The optimum parameters of amplitudes of excitation and positions obtained by our formulation using PSO can be utilizing to plot the variation of radiation patterns of linear array of $(1,8,10$ and 16) elements, which shown in Figures 5-8. Clearly, our results are generally better in terms of the sidelobe level and directivity of radiation pattern than those suggested by using Dolph-chebyshev distribution. This last distribution is considered as the optimal array for the uniform array, but the Dolph-Chebychev distribution also has uniform sidelobes. However, for some applications, a non-uniforme array is desirable. For the case of array of rectangular superconducting microstrips with multilayer dielectrics, the radiation patterns is calculated by using the product of the element factor Eq ,F. and the array factor $\operatorname{AF}(q, f)$.

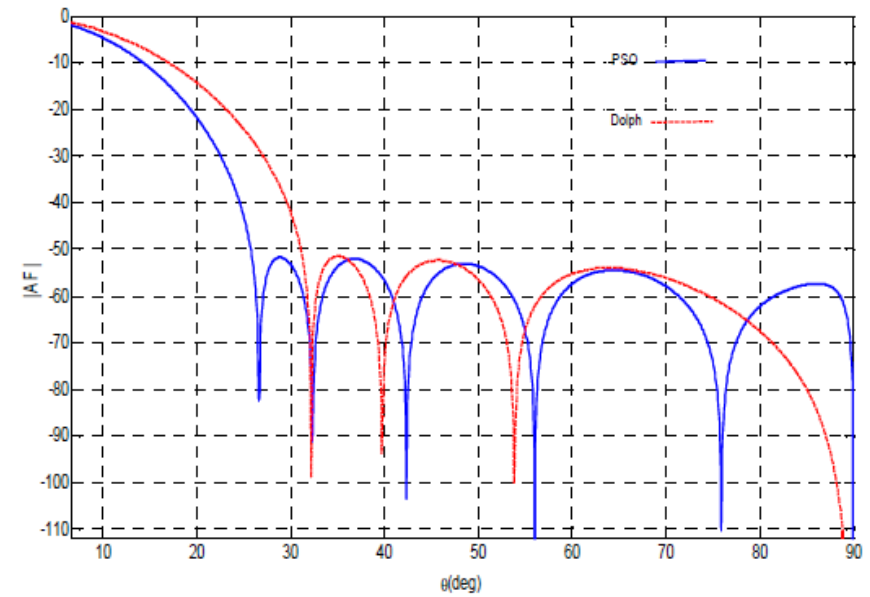

Fig. 4. Radiation pattern of linear array of 8 rectangular superconducting microstrip antenna versus angle $\theta$ 


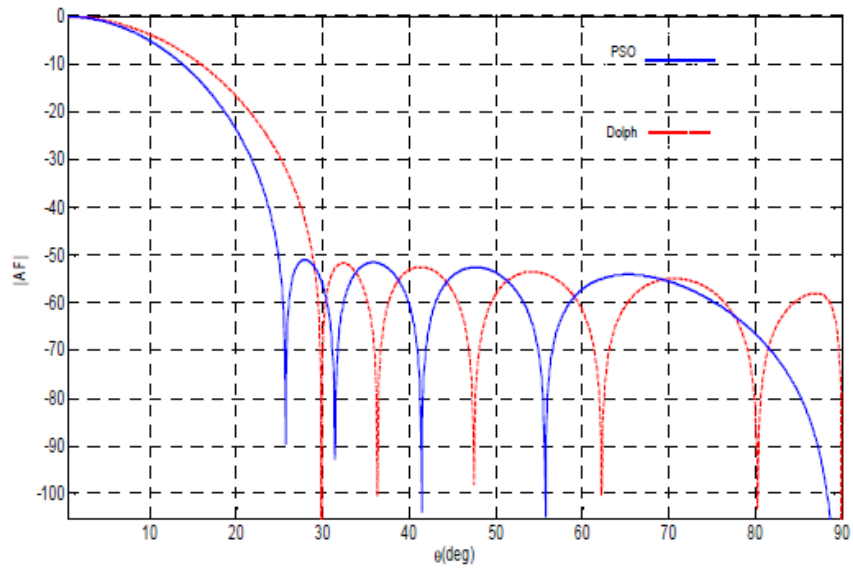

Fig. 5. Radiation pattern of linear array of 10 rectangular superconducting microstrip antenna versus angle $\theta$

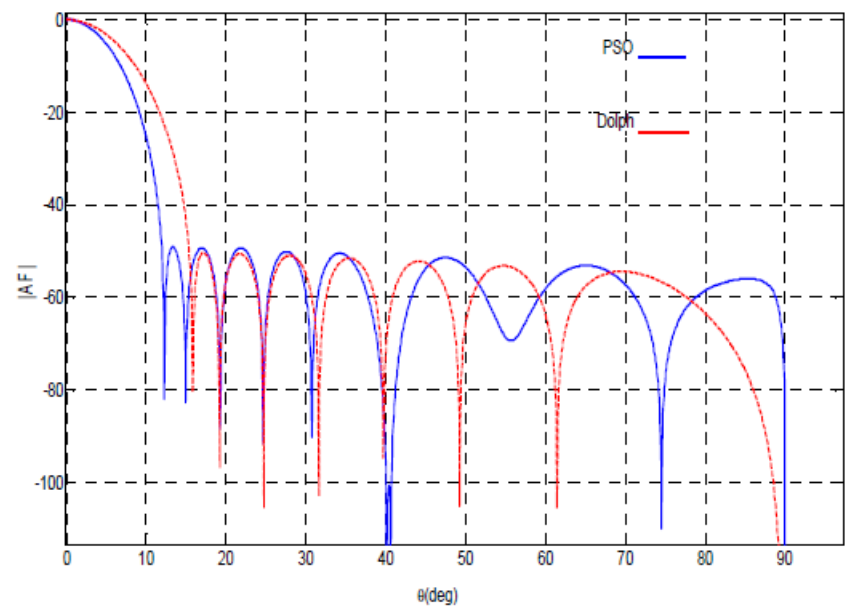

Fig. 6. Radiation pattern of linear array of 16 rectangular superconducting microstrip antenna versus angle $\theta$

\section{CONCLUSION}

The spectral domain and the stationary phase formulations have been used for the numerical calculation of the resonant frequency and radiated field of a of single rectangular microstrip antenna. The resolution of the integral equations of the electric field by the procedure of Galerkin makes it possible to lead to a system of equations homogenous. Variation of the resonant frequency versus the thickness of High superconducting rectangular microstrip on uniaxial substrate has been presented. In addition, we have presented an optimization technique based on particles swarm optimization algorithm, which is successfully used to determine the element excitations and positions of non-uniform linear antenna arrays, for simultaneous reduction of the side lobe level. The far field radiation patterns obtained by PSO algorithm are compared with the far field radiation patterns by Dolph-chebyshev distribution. A comparative study shows that PSO is capable of synthesising the unequally spaced linear antenna arrays to produce radiation patterns with a good performance in the sidelobe region.

\section{REFERENCES}

[1] C. A. Balanis, Antenna theory: analysis and design. John Wiley and Sons, Hoboken, NJ, USA, 2005.

[2] J. R. James, P. S. Hall and C. Wood, Microstrip antenna theory and design, 1981. https://doi.org/10.1049/PBEW012E

[3] L. H. Randy Antenna arrays: a computational approach. John Wiley \& Sons, Inc, Hoboken, New Jersey, 2010.

[4] M. A. Richard, K. B. Bhasin, P. C. Claspy, "Superconducting microstrip antennasd: An experimental comparison of two feeding methods. IEEE Trans. Antennas Propagat;1993. Vol 41. ํ⒎ p. 967-974. https://doi.org/10.1109/8.237630

[5] R. C. Hansen Electrically Small, Superdirective, and Superconducting Antennas, John Wiley\& Sons, Inc, Hoboken, New Jersey; 2006. https://doi.org/10.1002/0470041048

[6] O. Barkat, A. Benghalia , "Radiation and resonant frequency of superconducting annular ring microstrip antenna on uniaxial anisotropic media," Springer, Journal of Infrared, Millimeter, and Terahertz Waves, vol 30, No 10, pp. 1053-1066, 2009.

https://doi.org/10.1007/s10762-009-9526-2

[7] Z. Cai, J. Bornemann, "Generalized Spectral Domain Analysis for Multilayered Complex Media and High Tc Superconductor application," IEEE Transactions on microwave Theory and Techniques, vol 40, $\mathrm{N}^{\circ} 12$, pp. 2251- 2257, 1992. https://doi.org/10.1109/22.179887

[8] O. Barkat, "Modeling and optimization of radiation characteristics of triangular superconducting microstrip antenna array," Journal of Computational Electronics, Springer, vol 13. N³. pp. 657-665, 2014. https://doi.org/10.1007/s10825-014-0584-x

[9] SO. Park, CA. Balanis, CR. Birtcher, "Analytical evaluation of the asymptotic impedance matrix of a grounded dielectric slab with roof top functions," IEEE Trans Antennas Propagat, vol 46. $\mathrm{N}^{\circ}$ 2. pp.251-259, 1998. https://doi.org/10.1109/8.660970

[10] J. R. Mohammed, "Phased Array Antenna with Ultra-Low Sidelobes," Electronics Letters, vol 49, issue 17, pp. 1055-1056, August 2013. https://doi.org/10.1049/el.2013.1642

[11] J. R. Mohammed, "Optimal Null Steering Method in Uniformly Excited Equally Spaced Linear Array by Optimizing Two Edge Elements," Electronics Letters, vol 53, issue 11, 2017. https://doi.org/10.1049/el.2017.1405

[12] J. R. Mohammed, and K. H. Sayidmarie, "A Null Steering Method by Controlling Two Elements," IET Microwaves, Antennas \& Propagation, vol 8, issue 15, pp.1348-1355, 2014. https://doi.org/10.1049/iet-map.2014.0213

[13] O. Barkat, "Theoretical study of superconducting annular ring microstrip antenna with several dielectric layers," Progress in electromagnetics research, vol 127, pp. 31-48. 2012. https://doi.org/10.2528/PIER12020105

[14] F. Abboud, J. P. Damiano, A. Papiernik, "Accurate model for the input impedance of coax-fed rectangular microstrip antenna with and without air gaps," Proceedings of Sixth International Conference on Antennas and Propagation, vol 1, pp. 102-106, April 1989. 